\title{
Corrections to the paper ' On orbits of unipotent flows on homogeneous spaces'
}

\author{
S. G. DAN I \\ School of Mathematics, Tata Institute of Fundamental Research, Homi Bhaba Road, \\ Bombay 400 005, India \\ (Received 8 January 1986)
}

The author regrets that there are certain errors in [1] and would like to give the following corrections.

(1) The last part of the statement of lemma 2.5 should be 'if and only if $\rho\left(u_{t} g\right) v=$ $\rho(g) v$ for all $t \in \mathbb{R}^{\prime}$, which is what the proof actually shows. In the proof of corollary 2.6, applying the corrected version of lemma 2.5 leads to ' $u_{\imath} g \gamma \in g \gamma \sigma^{-1}(K \cap Z) \cdot N \sigma$ for all $t \in \mathbb{R}^{\prime}$ in place of the displayed line. The validity of the corollary is unaffected.

(2) In the proof of theorem 4.1 the assertion that 'we may assume $\Gamma$ to be an irreducible lattice' is not valid; the author is thankful to $D$. Witte for pointing this out. The error, however, can be easily rectified using the full strength of theorem 0.2. In any case, a variation of the proof of theorem 3.5 in [2], involving theorem 3.2 in the place of theorem 3.1 , implies a stronger result.

\section{REFERENCES}

[1] S. G. Dani. On orbits of unipotent flows on homogeneous spaces. Ergod. Th. \& Dynam. Sys. 4 (1984), 25-34.

[2] S. G. Dani. On orbits of unipotent flows on homogeneous spaces, II. Ergod. Th. \& Dynam. Sys. 6 (1986), 167-182. 